\title{
Analysis and Optimization of Micro Speaker-Box Using a Passive Radiator in Portable Device
}

\author{
Yuan-Wu JIANG(1), Joong-Hak KWON(2), Hyung-Kyu KIM ${ }^{(1)}$, Sang-Moon HWANG(1) \\ (1) School of Mechanical Engineering \\ Pusan National University \\ Busan 609-735, Korea; e-mail: shwang@pusan.ac.kr \\ (2) Research and Development Center, EM-Tech \\ Changwon 642-120, Korea; e-mail: jh.kwon@em-tech.co.kr
}

(received September 8, 2016; accepted May 3, 2017)

\begin{abstract}
With the rapid development of multimedia devices such as smart phones and tablet PCs, microspeakers have been recently increasingly used for audio equipment. Improving the acoustic performance of a microspeaker is always a main concern, especially in the low frequency range. To avoid sound cancelation, a microspeaker unit is usually inserted into a speaker box. A passive radiator is also used in speaker boxes to improve the sound performance in the loudspeaker system. However, passive radiators have not been applied into microspeaker system. In this study, a speaker box with a passive radiator was analyzed and optimized to achieve a higher Sound Pressure Level (SPL) in a microspeaker system. The Finite Element Method (FEM), two-degree-of-freedom (DOF) vibration theory, and a plane circular piston sound source were used to study the electromagnetic, vibration, and acoustic characteristics, respectively. Optimization was conducted by changing the mass, stiffness, and size of the passive radiator. Based on the optimized parameters, a new sample was manufactured. The experiment results show that the SPL of the optimized speaker box with a passive radiator is improved by $5 \mathrm{~dB}$ at $200 \mathrm{~Hz}$ compared with the one without a radiator. The analysis results also matched the experiment results.
\end{abstract}

Keywords: passive radiator; speaker box; SPL improvement.

\section{Introduction}

The mobile communication industry supported by information and electronics technology is changing the way for human communication. With the wide use of multimedia mobile devices, microspeakers need to provide better performance as audio equipmen. A microspeaker unit is usually designed in a speaker box to avoid sound cancelation. Previous research utilized one-degree-of-freedom (DOF) vibration theory and considered the added stiffness of the speaker box (XU, 2014; Sun, 2014). However, a passive radiator was not included. A passive radiator is used in a speaker box to improve sound performance in a loudspeaker system, which is studied using an equivalent electrical circuit method (Leach, 2003; SMall, 1974a; 1974b). However, the same procedure cannot be applied to microspeakers due to the size difference. In this study, an analysis method for a speaker box using a passive radiator was developed and experimentally verified. Base on the analysis method, optimization was conducted to improve acoustic performance.

\section{Modelling}

The working principle of a speaker box with a passive radiator is illustrated in Fig. 1. The coil of a mi-

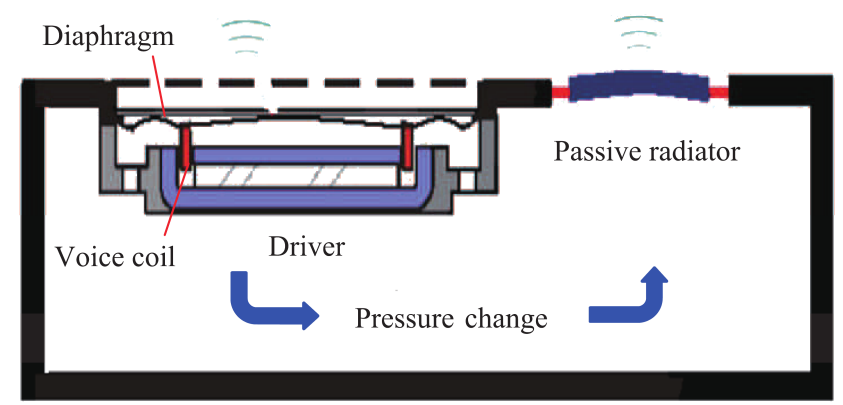

Fig. 1. Working principle of speaker box with a passive radiator. 
crospeaker unit or driver is excited by the Lorentz force, which pushes the diaphragm of the driver. The air in front and back of the diaphragm is compressed by the vibrated diaphragm. Thus, the air in the back volume is changed because of the air pressure variation. The air pressure change results in vibration of the radiator, which is why it is called a passive radiator.

Even though there is no real spring between the driver and the radiator, the effect can be considered as an "air spring". Therefore, the speaker box with a passive radiator can be modelled using 2 -DOF vibration, which is shown in Fig. 2.

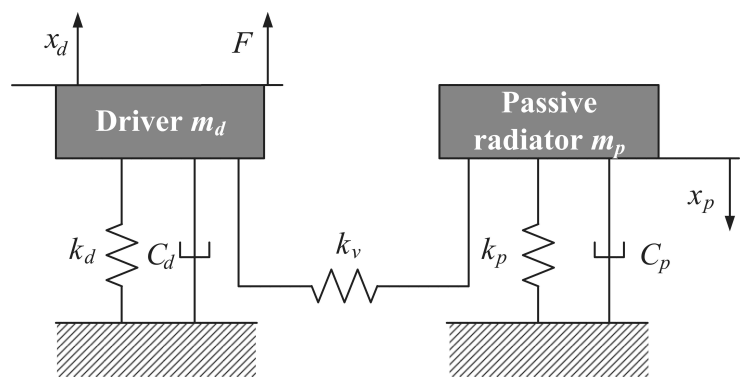

Fig. 2. 2-DOF modeling.

The governing equation is:

$$
\begin{aligned}
& m_{d} \ddot{x}_{d}+k_{d} x_{d}+C_{d} \dot{x}_{d}+\frac{\rho_{0} c^{2} S_{d}^{2}}{V_{c c}} x_{d} \\
&-\frac{\rho_{0} c^{2} S_{d} S_{p}}{V_{c c}} x_{p}=F \mathrm{e}^{\mathrm{j} w t}, \\
& m_{p} \ddot{x}_{p}+k_{p} x_{p}+C_{p} \dot{x}_{p}+\frac{\rho_{0} c^{2} S_{p}^{2}}{V_{c c}} x_{p} \\
&-\frac{\rho_{0} c^{2} S_{d} S_{p}}{V_{c c}} x_{d}=0,
\end{aligned}
$$

where

$$
x_{d}=\left|x_{d}\right| \mathrm{e}^{\mathrm{j} w t+\theta_{d}}, \quad x_{p}=\left|x_{p}\right| \mathrm{e}^{\mathrm{j} w t+\theta_{p}} .
$$

In these equations, $m_{d}, k_{d}, C_{d}, S_{d}$ and $x_{d}$ are the mass, stiffness, damping, effective area, and displacement of vibration part the driver (microspeaker unit), while $m_{p}, k_{p}, C_{p}, S_{p}$ and $x_{p}$ are equivalent values for the passive radiator, respectively. $F, \rho_{0}, c, V_{c c}$ and $w$ are the Lorentz force, air density, air speed, back volume in the speaker box, and angular frequency.

After solving the 2-DOF vibration equations (RAO, 2004 ), the amplitude and phase information of displacement can be obtained:

$$
\begin{array}{ll}
\left|x_{d}\right|=\frac{F \sqrt{a_{9}^{2}+a_{10}^{2}}}{a_{7}^{2}+a_{8}^{2}}, & \theta_{d}=\tan ^{-1}\left(\frac{a_{10}}{a_{9}}\right), \\
\left|x_{p}\right|=\frac{F\left|a_{4}\right| \sqrt{a_{7}^{2}+a_{8}^{2}}}{a_{7}^{2}+a_{8}^{2}}, & \theta_{p}=\tan ^{-1}\left(\frac{-a_{8}}{a_{7}}\right),
\end{array}
$$

where $\left|x_{d}\right|$ and $\theta_{d}$ are the displacement amplitude and phase of the driver; while $\left|x_{p}\right|$ and $\theta_{p}$ are the displacement amplitude and phase of the passive radiator,

$$
\begin{aligned}
a_{1} & =-w^{2} m_{d}+k_{d}+k_{v 11}, \\
a_{2} & =w C_{d}, \\
a_{3} & =-k_{v 12}, \\
a_{4} & =-k_{v 21}, \\
a_{5} & =-w^{2} m_{p}+k_{p}+k_{v 22}, \\
a_{6} & =w C_{p}, \\
a_{7} & =a_{1} a_{5}-a_{2} a_{6}-a_{3} a_{4}, \\
a_{8} & =a_{2} a_{5}+a_{1} a_{6}, \\
a_{9} & =a_{5} a_{7}+a_{6} a_{8}, \\
a_{10} & =a_{6} a_{7}-a_{5} a_{8}, \\
k_{v 11} & =\frac{\rho_{0} c^{2} S_{d}^{2}}{V_{c c}}, \\
k_{v 12} & =\frac{\rho_{0} c^{2} S_{d} S_{p}}{V_{c c}}, \\
k_{v 22} & =\frac{\rho_{0} c^{2} S_{p}^{2}}{V_{c c}}, \\
k_{c c} & \rho_{0} c^{2} S_{d} S_{p} \\
k_{c c} &
\end{aligned}
$$

In acoustic analysis, the diaphragm is assumed to be a circular plane piston model with vibration in only the normal direction. The Sound Pressure Level (SPL) can be calculated by applying acoustics theory as follows (KINSLER, 1999):

$$
\left|p_{d}\right|=\frac{1}{2} \rho_{0}\left|x_{d}\right| w^{2} a^{2} / r,
$$

where $a$ is the effective radius value of piston sound source, $r$ is the distance between the sound source and measurement point, and $w$ is the angular frequency. In the same way, the sound pressure amplitude of the passive radiator is:

$$
\left|p_{p}\right|=\frac{1}{2} \rho_{0}\left|x_{p}\right| w^{2} a^{2} / r .
$$

The superposed sound pressure is depicted in Fig. 3 and can be calculated using Eq. (5). It was assumed that the driver and passive radiator are at the same position (SMALL, 1974a; 1974b)

$$
p_{\text {total }}=\left|p_{d}\right| \mathrm{e}^{\mathrm{j}\left(w t+\beta_{d}\right)}-\left|p_{p}\right| \mathrm{e}^{\mathrm{j}\left(w t+\beta_{p}\right)},
$$

where

$$
\begin{aligned}
& \left|p_{d}\right| \mathrm{e}^{\mathrm{j}\left(w t+\beta_{d}\right)}=\left|p_{d}\right| \cos \left(w t+\beta_{d}\right)+\mathrm{j}\left|p_{d}\right| \sin \left(w t+\beta_{d}\right), \\
& \left|p_{p}\right| \mathrm{e}^{\mathrm{j}\left(w t+\beta_{p}\right)}=\left|p_{p}\right| \cos \left(w t+\beta_{p}\right)+\mathrm{j}\left|p_{p}\right| \sin \left(w t+\beta_{p}\right) .
\end{aligned}
$$




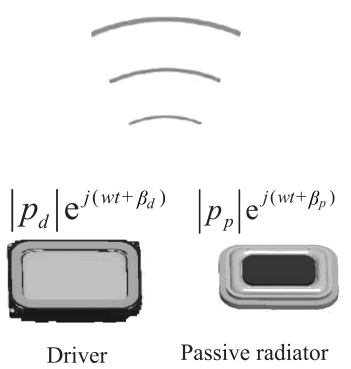

Fig. 3. Superposed sound pressure.

Finally, the total sound pressure can be calculated using Eq. (6):

$$
\left|p_{\text {total }}\right|=\sqrt{\left|p_{d}\right|^{2}+\left|p_{p}\right|^{2}+2\left|p_{d}\right| \cdot\left|p_{p}\right| \cos \left(\beta_{p}-\beta_{d}\right)},
$$

where

$$
\Delta \beta=\beta_{p}-\beta_{d}=\theta_{p}-\theta_{d}
$$

is the phase difference between the driver and passive radiator. SPL is calculated by:

$$
\operatorname{SPL}[\mathrm{dB}]=20 \log \frac{\left|p_{\text {total }}\right|}{\left|p_{0}\right|} .
$$

\section{Parameter identification}

Figure 4 shows an outline of the three types of speaker box systems. In order to apply the analysis method and optimize the speaker box, the parameters of the governing equations for type $\mathrm{A}$ and $\mathrm{B}$ should be identified. The mass of the vibration system is identified by an electronic scale, while the stiffness and damping are obtained by comparing the analysis and experiment results. The effective area is identified by Klippel equipment, and the magnetic force is obtained by the electromagnetic Finite Element Method (FEM).

a)

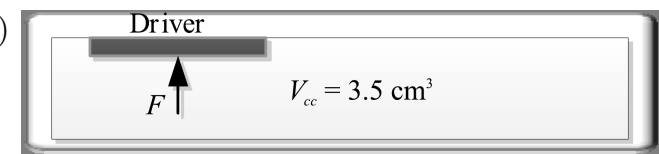

Passive radiator

b)

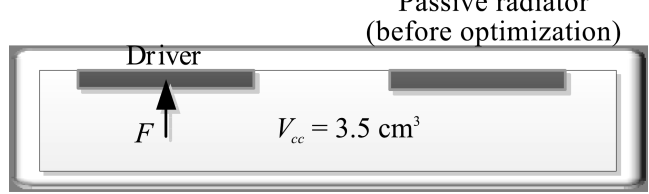

Passive radiator

c)

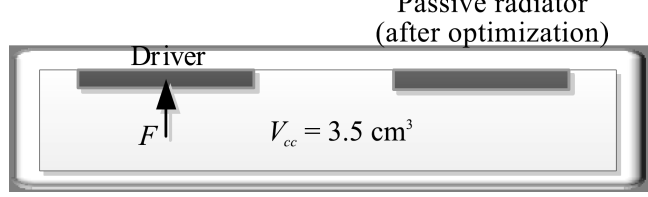

Fig. 4. Speaker box system: a) type A, b) type B, c) type C.

\subsection{Mass identification}

The mass can be obtained by an electronic scale (HR-200). The vibration parts of the passive radiator and driver are shown in Fig. 5. In Fig. 5 CDP represents centre diaphragm. It is attached on the centre of diaphragm, with material of aluminum. CDP can ensure that the diaphragm vibrates as a rigid body.

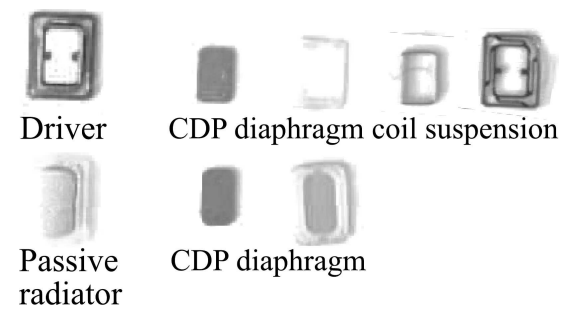

Fig. 5. Vibration parts of the passive radiator and driver.

\subsection{Stiffness and damping identification}

The stiffness and damping are not measured directly but are identified by comparing the analysis results from 2-DOF theory with the experiment results. The Klippel experiment equipment is depicted in Fig. 6. A laser detects the displacement of the vibration part of the driver.

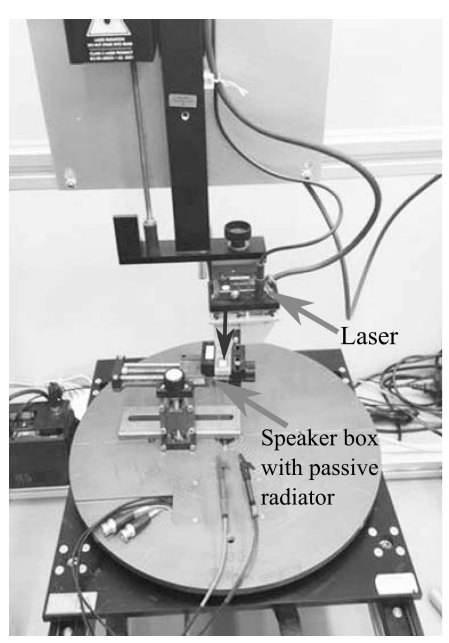

Fig. 6. Klippel equipment setup.

The comparison of the experiment and analysis results is shown in Fig. 7. After the stiffness and damping change, the analysis results of displacement change correspondingly. The parameters are determined as the values that make the analysis results approach the experiment results. The following results were obtained:

$$
\begin{array}{ll}
k_{d}=900 \mathrm{~N} / \mathrm{m}, & C_{d}=0.22 \mathrm{~N} \cdot \mathrm{s} / \mathrm{m}, \\
k_{p}=3600 \mathrm{~N} / \mathrm{m}, & C_{p}=0.12 \mathrm{~N} \cdot \mathrm{s} / \mathrm{m}
\end{array}
$$


a)

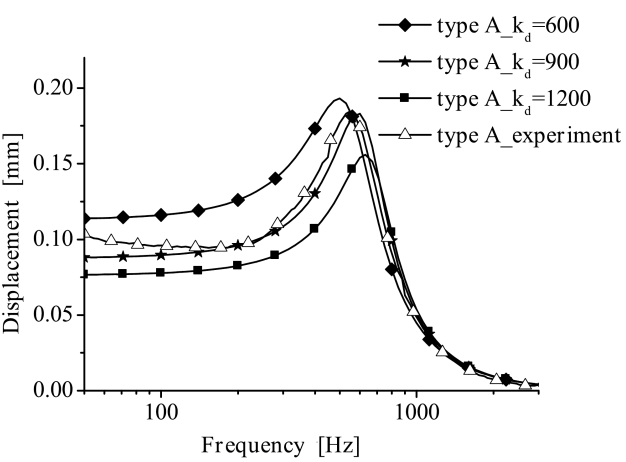

c)

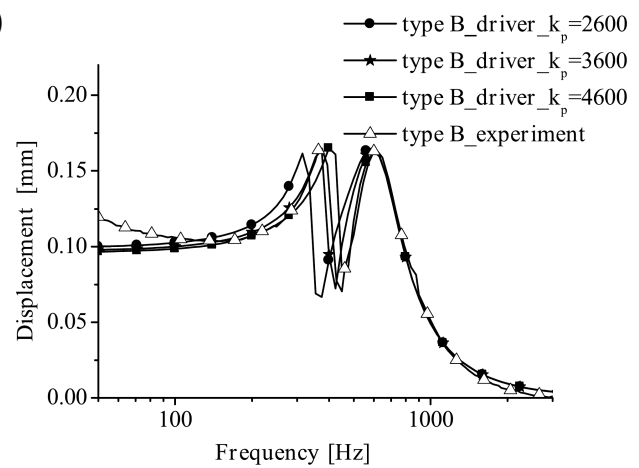

b)

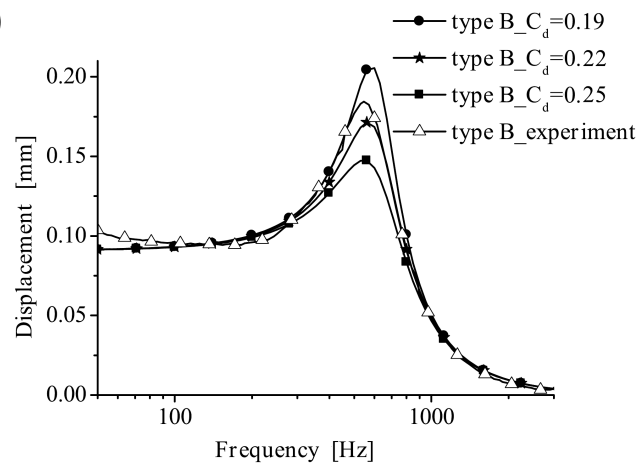

d)

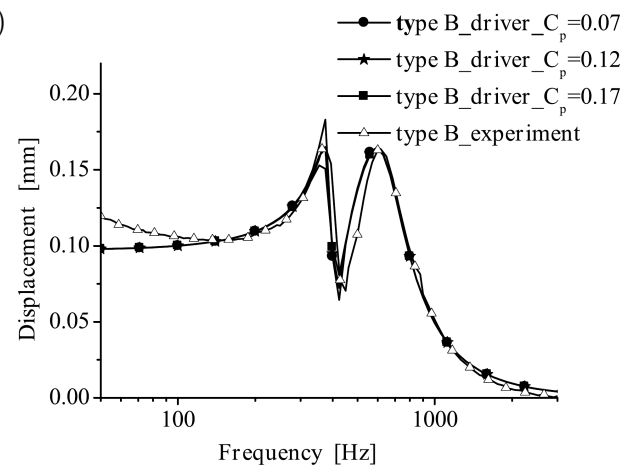

Fig. 7. Parameter identification: a) $k_{d}$ identification, b) $C_{d}$ identification, c) $k_{p}$ identification, d) $C_{p}$ identification.

\subsection{Effective area identification}

The identification method of effective area is shown in Fig. 8 (KLIPPEL, 2010). Considering the same complex volume velocity, the effective area is calculated using equation

$$
\underline{S}_{D}(w)=\frac{\underline{V}(w)}{j w \underline{x}_{\mathrm{coil}}(w)}=\frac{\int_{S c} \underline{x}\left(w, r_{c}\right) \mathrm{d} S}{\underline{x}_{\mathrm{coil}}(w)},
$$

where $\underline{x}\left(w, r_{c}\right)$ is the displacement of a point on the diaphragm, $r_{c}$ is the distance between the point and the center of the diaphragm, $\underline{V}(w)$ is the complex volume velocity, $\underline{x}_{\text {coil }}(w)$ is the average displacement of the point on the coil, and $w$ is the angular frequency.

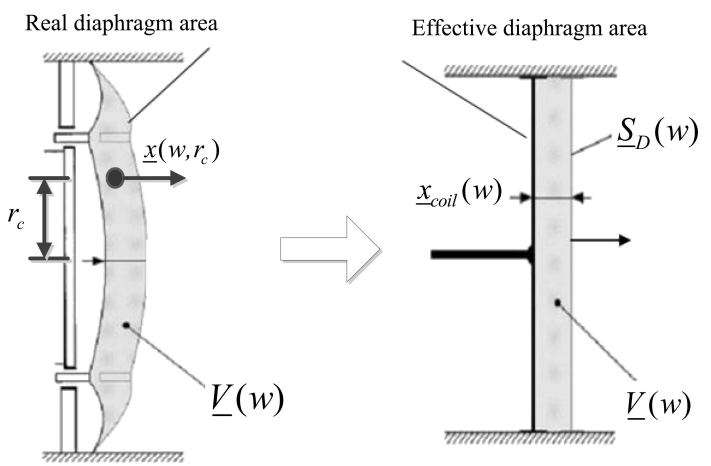

Fig. 8. Effective area calculation.
The calculation method for $\underline{x}_{\text {coil }}(w)$ and $\int_{S_{c}} \underline{x}\left(w, r_{c}\right) \mathrm{d} S$ is shown in Fig. 9. For calculating $\int_{S c} \underline{x}\left(w, r_{c}\right) \mathrm{d} S$, the equipment separates the diaphragm into finite elements, in which the displacement of a point represents the displacement of all points in the element.

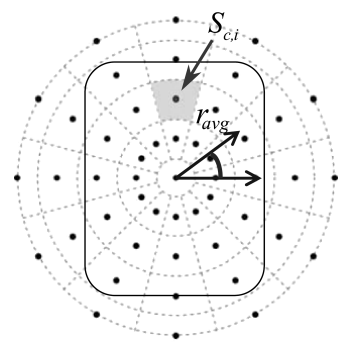

Fig. 9. Diaphragm calculation method.

The equations can be expressed as

$$
\begin{aligned}
\int_{S c} \underline{x}\left(w, r_{c}\right) \mathrm{d} S= & \sum \underline{x}\left(w, r_{c, i}\right) \cdot \Delta S_{c, i}, \\
\underline{x}_{\text {coil }}(w) & =\frac{\int_{0}^{2 \pi} \underline{x}\left(w, r_{\mathrm{avg}}, \varphi\right) \mathrm{d} \varphi}{2 \pi} .
\end{aligned}
$$

The effective area can be measured by replacing the speaker box with a microspeaker unit, as shown in Fig. 6. 


\subsection{Force identification}

The magnetic circuit of the microspeaker consists of a voice coil, top plate, permanent magnet, and yoke as shown in Fig. 10. An electromagnetic Finite Element Method (FEM) is used to obtain the average flux density of the voice coil (PAUL, 2001).

$$
\frac{\partial}{\partial x}\left(\frac{1}{\mu} \frac{\partial A}{\partial x}\right)+\frac{\partial}{\partial y}\left(\frac{1}{\mu} \frac{\partial A}{\partial y}\right)=J_{0}-\frac{1}{\mu}\left(\frac{\partial M_{y}}{\partial x}-\frac{\partial M_{x}}{\partial y}\right)
$$

where $A$ is the magnetic potential, is the magnetic permeability, $J_{0}$ is the current density, and $M$ is the magnetic moment. After the material properties are input and the vector potential of the model boundary is defined as zero, the magnetic flux density can be calculated. The average magnetic flux density at the initial coil position is $0.544 \mathrm{~T}$. The microspeaker is operated with an input of $0.1 W_{\mathrm{rms}}$. The magnetic force is calculated by

$$
F_{\mathrm{mag}}=\oint I \mathrm{~d} l \times B
$$

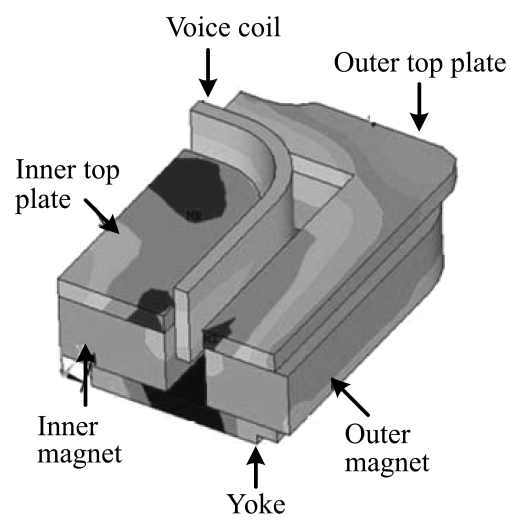

Fig. 10. Electromagnetic analysis.

To ensure that the electromagnetic FEM is correct, Klippel equipment is used to measure force factor $\mathrm{Bl}$. As shown in Fig. 6, the force factor can be measured by replacing the speaker box with a microspeaker unit. The difference between the value of $\mathrm{Bl}$ calculated using the electromagnetic FEM $(0.824 \mathrm{~N} / \mathrm{A})$ and the measured value of $\mathrm{Bl}(0.820 \mathrm{~N} / \mathrm{A})$ is less than $1 \%$.

The identified parameters of the speaker box with a passive radiator are listed in Table 1 . After the parameters are substituted into Eq. (7), the analysis re-

Table 1. Parameters of speaker box with passive radiator.

\begin{tabular}{|c|c|c|c|c|c|}
\hline \multicolumn{2}{|c|}{ Microspeaker driver } & \multicolumn{3}{c|}{ Passive radiator (prototype) } \\
\hline $\mathbf{F}$ & 99.5 & $\mathrm{~m} \cdot \mathrm{N}$ & $V_{c c}$ & 3.5 & $\mathrm{cc}$ \\
\hline$m_{d}$ & 103 & $\mathrm{mg}$ & $m_{p}$ & 642.71 & $\mathrm{mg}$ \\
\hline$k_{d}$ & 900 & $\mathrm{~N} / \mathrm{m}$ & $k_{p}$ & 3600 & $\mathrm{~N} / \mathrm{m}$ \\
\hline$C_{d}$ & 0.22 & $(\mathrm{~N} \cdot \mathrm{s}) / \mathrm{m}$ & $C_{p}$ & 0.12 & $(\mathrm{~N} \cdot \mathrm{s}) / \mathrm{m}$ \\
\hline$S_{d}$ & 118 & $\mathrm{~mm}^{2}$ & $S_{p}$ & 126 & $\mathrm{~mm}^{2}$ \\
\hline
\end{tabular}

sults of SPL can be achieved using the method developed in modelling. Figures 11 and 12 depict the comparison of SPL between the experimental result and analysis result for types $\mathrm{A}$ and $\mathrm{B}$. In the frequency range of $200 \mathrm{~Hz}$ to $3 \mathrm{kHz}$, the difference between simulation and experimental results are within $1.5 \%$. In addition, above $3 \mathrm{kHz}$, the analysis results do not match the experiment results. The reason is that $2-\mathrm{DOF}$ vibration theory does not consider higher modes, and sound reflection is ignored. Therefore, the analysis tool can be applied at low frequency, which is the focus of this paper.

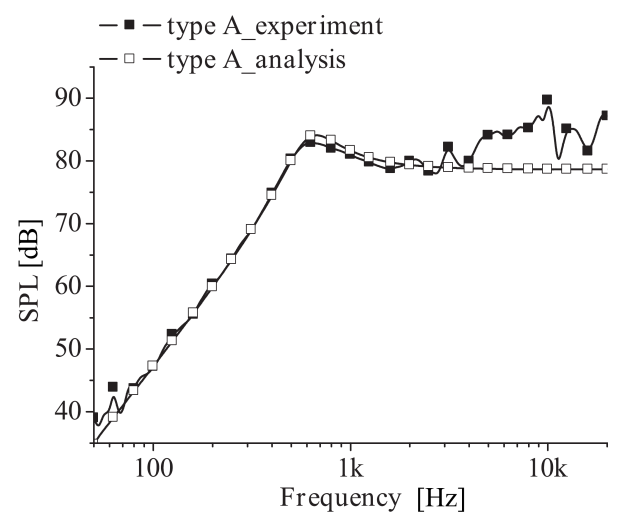

Fig. 11. Analysis and experiment results (type A).

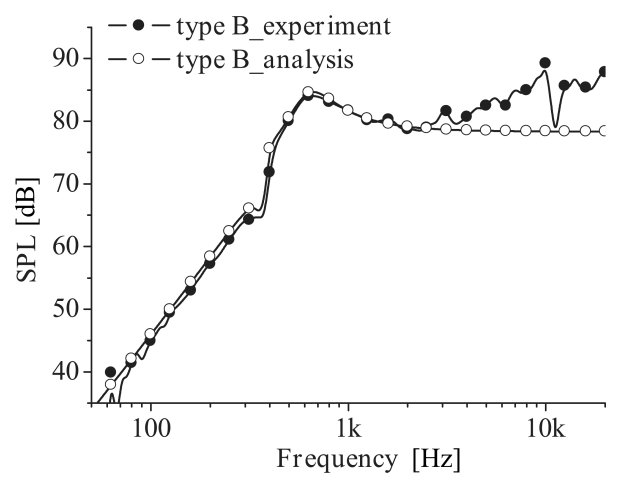

Fig. 12. Analysis and experiment results (type B).

In addition, the experiment results of types $\mathrm{A}$ and $\mathrm{B}$ were compared as shown in Fig. 13. The results

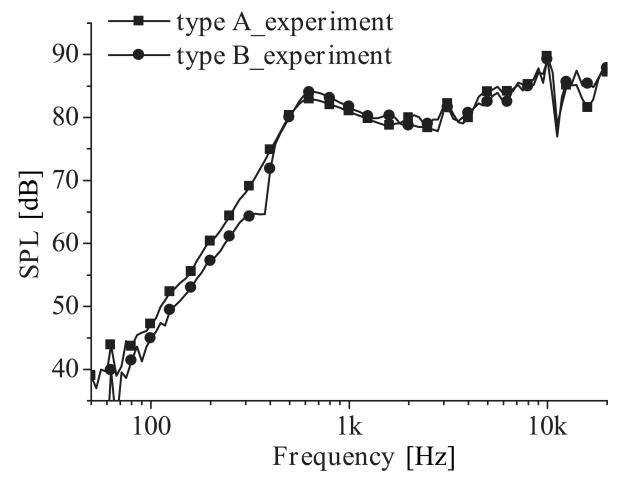

Fig. 13. Experimental results (type A and B). 
reveal that type B does not show SPL improvement at low frequency, so optimization of the passive radiator is needed.

\section{Optimization}

Because the damping is difficult to control, $m_{p}, k_{p}$, and $S_{p}$ are chosen as the parameters to be optimized. According to the 2-DOF analysis tool, the objective function is defined as Eq. (14). Figure 14 demonstrates the definition of the objective function.

$$
F=\int_{f_{\text {crossover }}}^{f_{\text {resonance }}}\left[\mathrm{SPL}_{\text {type } \mathrm{C}}(f)-\mathrm{SPL}_{\text {type A }}(f)\right] \mathrm{d} f
$$

$\mathrm{SPL}_{\text {type } \mathrm{C}}(f)$ and $\mathrm{SPL}_{\text {type } \mathrm{A}}(f)$ are the SPLs of speaker boxes with and without the passive radiator, $f_{\text {crossover }}$ is the crossover point of $\mathrm{SPL}_{\text {type } \mathrm{C}}(f)$ and $\mathrm{SPL}_{\text {type } \mathrm{A}}(f)$, and $f_{\text {resonance }}$ is the resonance frequency of type A.

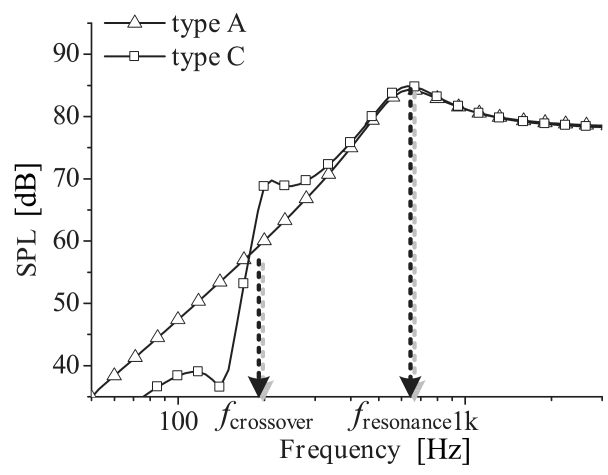

Fig. 14. Objective function definition.

The optimization method changes $m_{p}, k_{p}$, and $S_{p}$ within the physically possible range, which is shown in Table 2. Then, the SPL curve of type $\mathrm{C}$ can be generated, which means that the objective function can be calculated. Finally, the values of $m_{p}, k_{p}$, and $S_{p}$ that maximize the objective function are selected as the optimized values. Figure 16 shows the value of the objective function under different values of the passive radiator. According to the calculation, the maximum SPL difference can be obtained with the following: $m_{p}=300 \mathrm{mg}, k_{p}=100 \mathrm{~N} / \mathrm{m}, S_{p}=200 \mathrm{~mm}^{2}$. Using the optimized passive radiator, the SPL of type $\mathrm{C}$ can be depicted. The comparison of the analysis result is shown in Fig. 15. After the optimization, type C

Table 2. Parameter range.

\begin{tabular}{|c|c|c|c|}
\hline Item & $m_{p}[\mathrm{mg}]$ & $k_{p}[\mathrm{~N} / \mathrm{m}]$ & $S_{p}\left[\mathrm{~mm}^{2}\right]$ \\
\hline Maximum & 1000 & 900 & 200 \\
\hline Minimum & 200 & 100 & 120 \\
\hline Interval & 100 & 250 & 10 \\
\hline Level & 9 & 9 & 9 \\
\hline
\end{tabular}

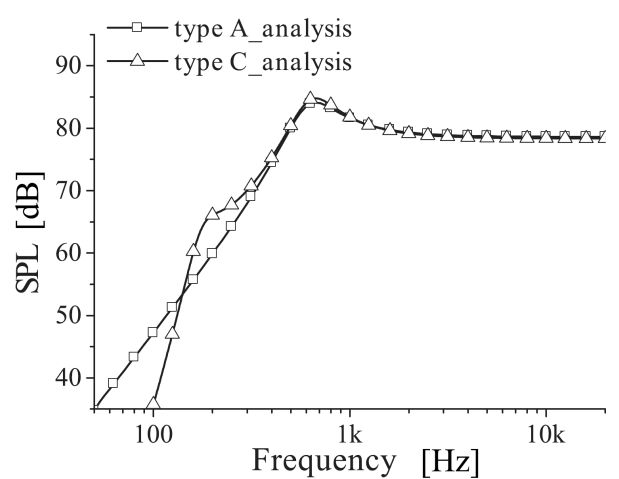

Fig. 15. Comparison of analysis results.

a)

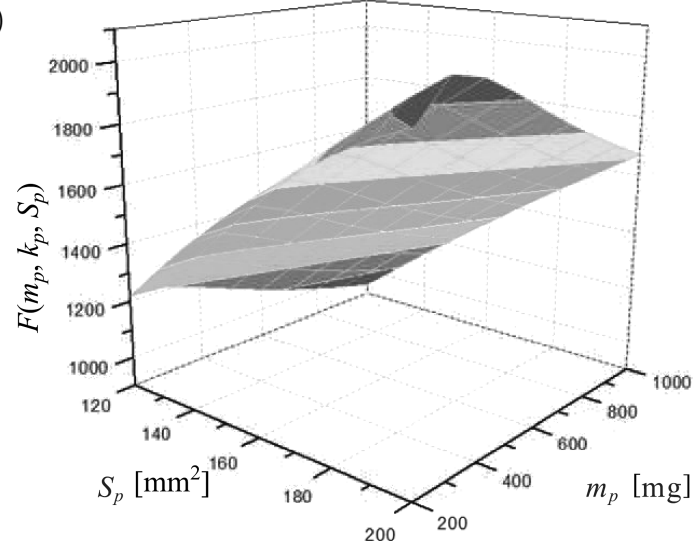

b)

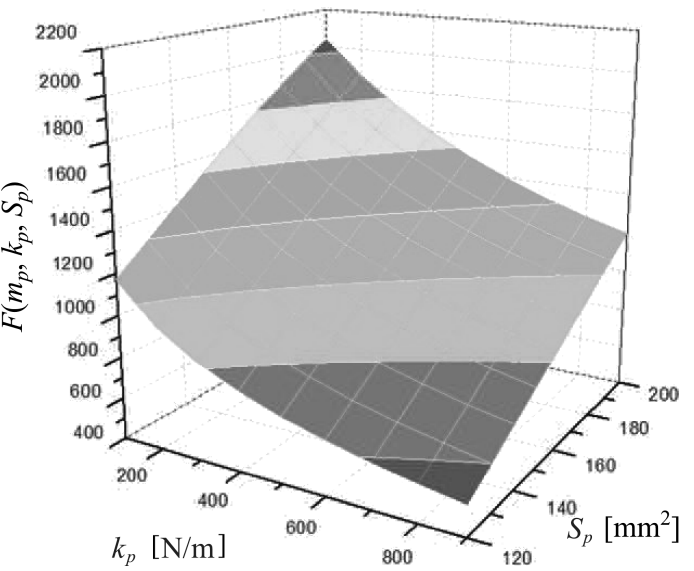

c)

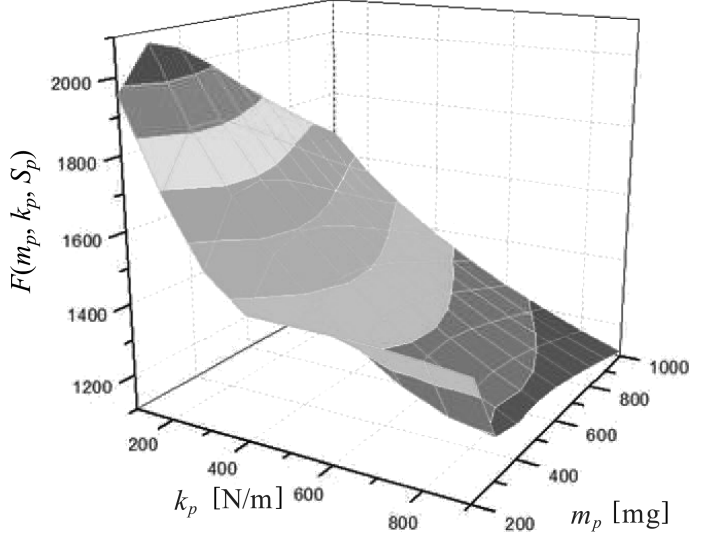

Fig. 16. Maximum of objective function: a) $k_{p}=100 \mathrm{~N} / \mathrm{m}$, b) $m_{p}=300 \mathrm{mg}$, c) $S_{p}=200 \mathrm{~mm}^{2}$. 
showed an obvious improvement at low frequency compared with type A. The main difference is that type $\mathrm{C}$ has a bigger radiator with very low stiffness.

\section{Experiment}

The optimized types were manufactured according to the design and optimization. The driver and passive radiator were assembled into speaker boxes, and three kinds of microspeaker systems were created, as shown in Fig. 17. Figure 18 shows the acoustic testing room and an outline of the connections of the testing devices. In the experiment, a B\&K Pulse system was applied. The testing frequency range was $50 \mathrm{~Hz}$ to $20 \mathrm{kHz}$. A swept sine signal was used. In the B\&K system, the received sound signal is transformed to the frequency domain by the Fast Fourier Transform (FFT). The testing distance was selected as $0.1 \mathrm{~m}$ in the central axial direction.

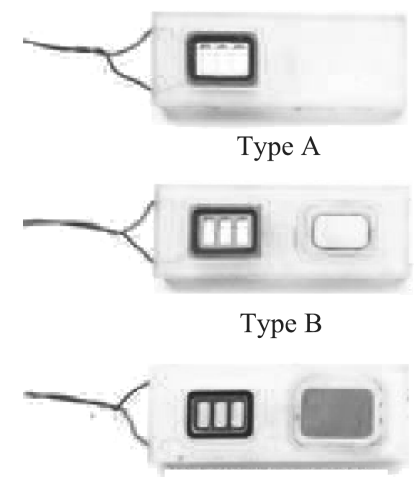

Type C

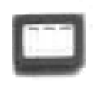

Driver

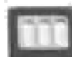

Passive radiator Driver (before optimization)

\section{UDE}

Driver Passive radiator (after optimization)
Fig. 17. Speaker box samples.

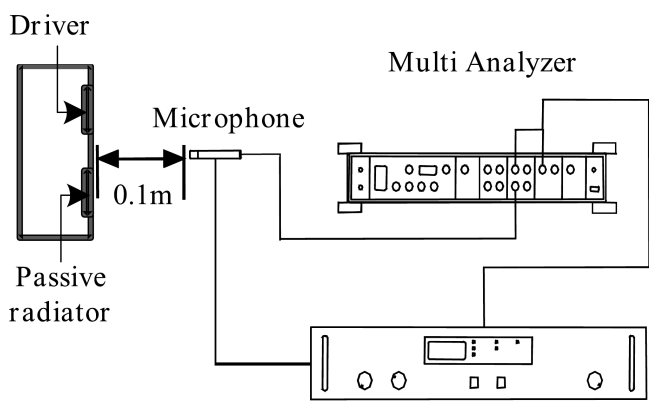

Power Amplifier

Fig. 18. Experiment setup.

Figure 19 shows a SPL comparison of the experiment and analysis results of type $\mathrm{C}$ and verifies the analysis method. Figure 20 shows a comparison of the experiment results of SPL between types A, B, and C. The experimental results reveal that type $\mathrm{C}$ has the best performance. Type $\mathrm{C}$ shows $5 \mathrm{~dB}$ improvement in the SPL at $200 \mathrm{~Hz}$ compared with type A.

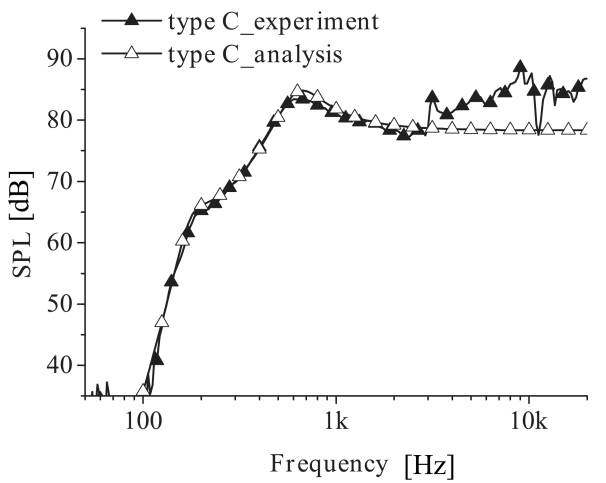

Fig. 19. Analysis and experiment results (type C).

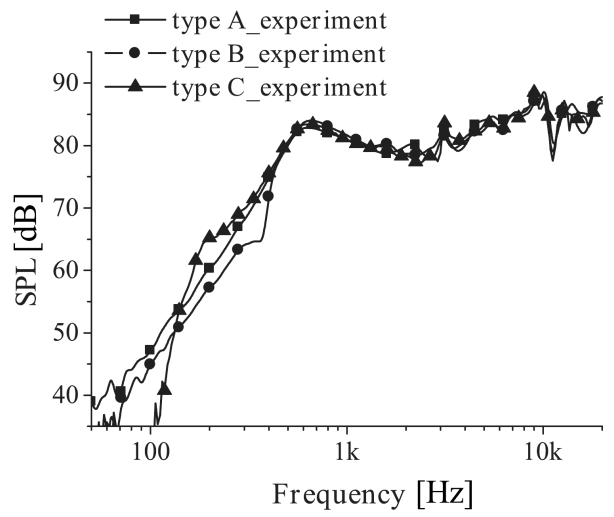

Fig. 20. Comparison of experiment results.

\section{Conclusion}

In this study, 2-DOF vibration theory was used to analyze a speaker box with a passive radiator. The parameters of the speaker box system were identified and optimized by changing the mass, stiffness, and area. Finally, speaker box samples were manufactured and tested. The analysis method was verified experimentally. As shown in Fig. 20, the optimized speaker box with a passive radiator (type $\mathrm{C}$ ) has the same resonance frequency $(630 \mathrm{~Hz})$ as the one without a passive radiator (type $\mathrm{A}$ ). Meanwhile, type $\mathrm{C}$ shows the $\mathrm{SPL}$ improvement from the crossover frequency $(150 \mathrm{~Hz})$ to the resonance frequency, compared with type A. Specifically, there is $5 \mathrm{~dB}$ improvement at $200 \mathrm{~Hz}$ and $2 \mathrm{~dB}$ improvement at $300 \mathrm{~Hz}$. In conclusion, a passive radiator can be used to improve the SPL in the low frequency domain.

\section{References}

1. Kinsler L.E., Frey A.R., Coppens A.B., Sanders J.V. (1999), Fundamentals of acoustics, 4th ed., Wiley$\mathrm{VCH}$.

2. Klippel W., Schlechter J. (2010), Dynamical measurement of the effective radiation area $S D$, Proceedings of 128th Audio Engineering Society Convention, London, Vol. 1, pp. 568-577. 
3. LEACH W.M. (2003), Introduction to electroacoustics and audio amplifier design, Kendall/Hunt Publishing Company.

4. Paul C.R., Nasar S.A. (2001), Introduction to electromagnetic fields, 3rd ed., McGraw-Hill.

5. Rao S.S. (2004), Mechanical vibrations, 4th ed., Pearson Education, Upper Saddle River.

6. Small R.H. (1974a), Passive-radiator loudspeaker systems. Part 1: Analysis, Journal of the Audio Engineering Society, 22, 592-601.
7. Small R.H. (1974b), Passive-radiator loudspeaker systems. Part 2: Synthesis, Journal of the Audio Engineering Society, 22, 683-689.

8. Sun P., Xu D.P., Hwang S.M. (2014), Design of microspeaker module considering added stiffness, Journal of Mechanical Science and Technology, 28, 5, 16231628.

9. Xu D.P., Sun P., Kwon J.H., Hwang S.M. (2014), An integrated design of microspeaker module with smaller volume, Journal of Applied Physics, 115, 17A339, doi: 10.1063/1.4868183. 